\title{
Quantifying Heartbeat Dynamics by Magnitude and Sign Correlations
}

\author{
Plamen Ch. Ivanov*, Yosef Ashkenazy ${ }^{\dagger}$, Jan W. Kantelhardt ${ }^{\dagger}$ and \\ H.Eugene Stanley ${ }^{\dagger}$ \\ ${ }^{*}$ Center for Polymer Studies and Department of Physics, Boston University, Boston, MA 02215 and \\ Beth Israel Deaconess Medical Center, Harvard Medical School, Boston, Massachusetts 02215, \\ USA \\ ${ }^{\dagger}$ Center for Polymer Studies and Department of Physics, Boston University, Boston, MA 02215
}

\begin{abstract}
We review a recently developed approach for analyzing time series with long-range correlations by decomposing the signal increment series into magnitude and sign series and analyzing their scaling properties. We show that time series with identical long-range correlations can exhibit different time organization for the magnitude and sign. We apply our approach to series of time intervals between consecutive heartbeats. Using the detrended fluctuation analysis method we find that the magnitude series is long-range correlated, while the sign series is anticorrelated and that both magnitude and sign series may have clinical applications. Further, we study the heartbeat magnitude and sign series during different sleep stages - light sleep, deep sleep, and REM sleep. For the heartbeat sign time series we find short-range anticorrelations, which are strong during deep sleep, weaker during light sleep and even weaker during REM sleep. In contrast, for the heartbeat magnitude time series we find long-range positive correlations, which are strong during REM sleep and weaker during light sleep. Thus, the sign and the magnitude series provide information which is also useful for distinguishing between different sleep stages.
\end{abstract}

A broad class of physical and biological systems exhibits complex dynamics, associated with the presence of many components interacting over a wide range of time or space scales. These often-competing interactions may generate an output signal with fluctuations that appear "noisy" and "erratic" but reveal scale-invariant structure. One general approach to study these systems is to analyze the ways that such fluctuations obey scaling laws $[1,2,3]$.

We consider the time series formed by consecutive cardiac interbeat intervals (Fig. 1a) and focus on the correlations in the time increments between consecutive beats. This time series is of general interest, in part because it is the output of a complex integrated control system, including competing stimuli from the neuroautonomic nervous system [4]. These stimuli modulate the rhythmicity of the heart's intrinsic pacemaker, leading to complex fluctuations. Previous reports indicate that these fluctuations exhibit scaleinvariant properties $[5,6,7]$, and are anticorrelated over a broad range of time scales (i.e., the power spectrum follows a power-law where the amplitudes of the higher frequencies are dominant) [8]. By long-range anticorrelations we also mean that the root mean square fluctuations function of the integrated series is proportional to $n^{\alpha}$ where $n$ is the window scale and the scaling exponent $\alpha$ is smaller than 0.5 . In contrast, for uncorrelated behavior $\alpha=0.5$, while for correlated behavior $\alpha>0.5$.

The time series of the fluctuations in heartbeat intervals can be "decomposed" into 

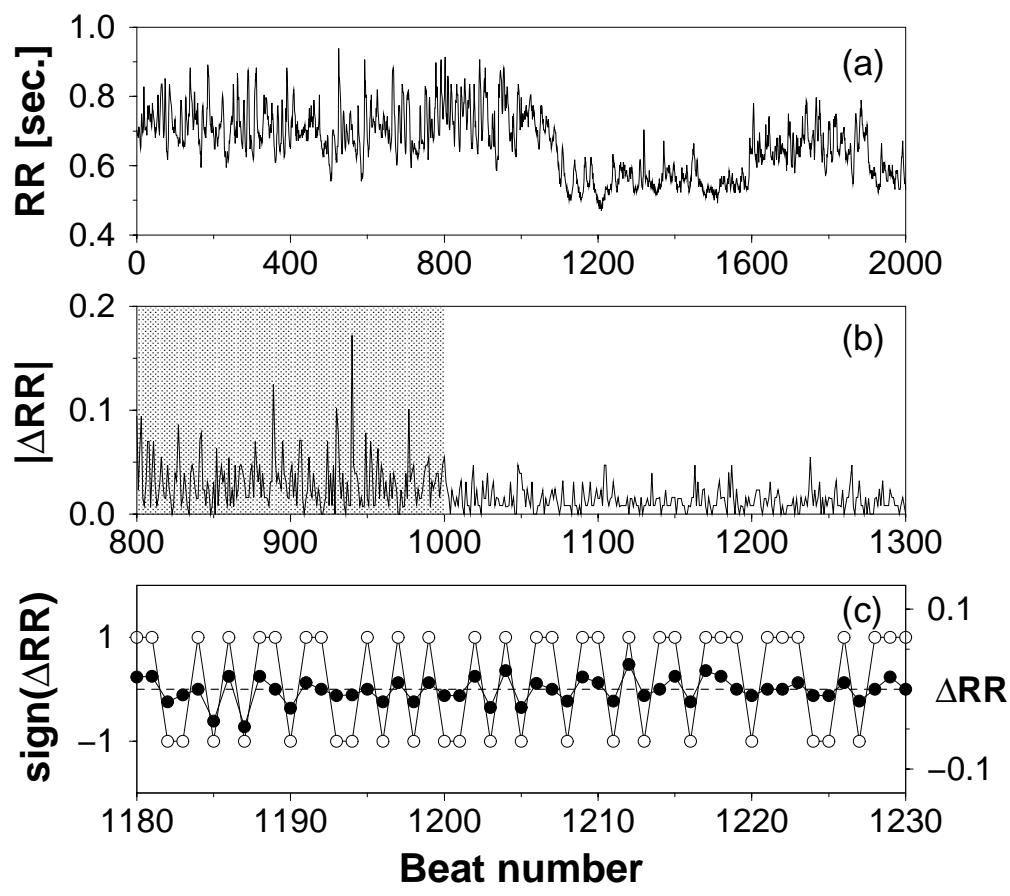

FIGURE 1. (a) An example of 2000 time intervals between successive $R$ peaks (RR intervals) taken from electrocardiogram (ECG) recording of a healthy subject during daytime. (b) The magnitude series of a portion of the RR series (beat numbers 800-1300) shown in (a). Patches of more "volatile" increments with large magnitude (beat numbers 800-1000) are followed by patches of less volatile increments with small magnitude (beat numbers 1000-1300), consistent with our quantitative conclusion that there is correlation in the magnitude time series. (c) The sign series (o), as well as the $\Delta R R$ series ( $\bullet$ ) of a portion of the RR series (beat numbers 1180-1230) shown in (a). The positive sign $(+1)$ represents a positive increment, while the negative sign $(-1)$ represents a negative increment in the RR series of interbeat intervals. The tendency to alternation between +1 and -1 is consistent with our quantitative conclusion that there is (multiscale) anticorrelation in the sign time series. Adapted from ref. [14].

two different time series. We analyze separately the time series formed by the magnitude and the sign of the increments in the time intervals between successive heartbeats (Fig. $1 \mathrm{~b}, \mathrm{c})$. We use 2 nd order detrended fluctuation analysis $[9,10,11,12]$ and not the conventional power spectrum, since it has the ability to accurately estimate correlations in the heartbeat fluctuations even when they are masked by linear trends. The 1st order detrended fluctuation analysis (DFA) eliminates constant trends from the original series (or, equivalently, linear trends from the integrated series); the 2nd order DFA removes linear trends, and the $k$ th order DFA eliminates polynomial trends of order $k-1$.

The magnitude/sign decomposition consists of the following steps: (i) given a series of successive interbeat intervals $R R_{i}$ we create the increment series, $\Delta R R_{i}=R R_{i+1}-R R_{i}$; (ii) we decompose the increment series into a magnitude series $(|\Delta R R|)$ and a sign series $(\operatorname{sign}(\Delta R R))$; (iii) to avoid artificial trends we subtract from the magnitude and sign series their average; (iv) because of limitations in the accuracy of the detrended 

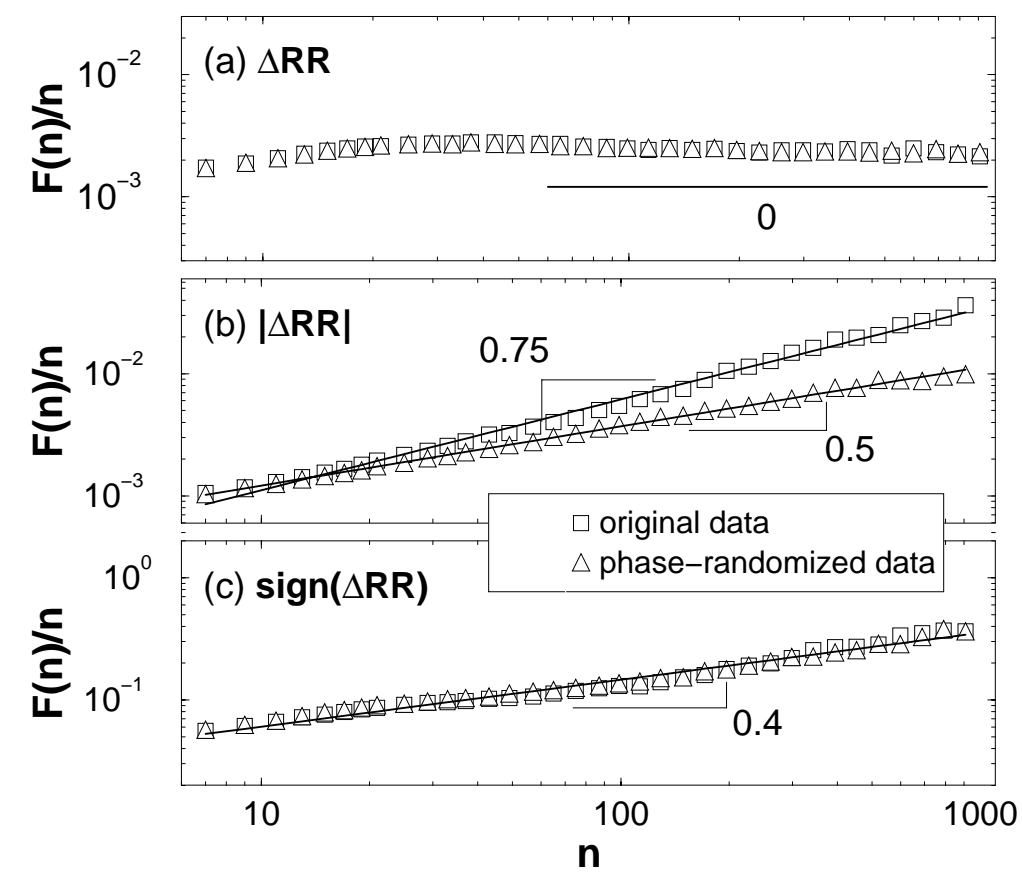

FIGURE 2. (a) Root mean square fluctuation, $F(n)$, for $\approx 6$ hour record $(\approx 32,000$ data points) for the interbeat interval $R R_{i}$ series ( $\square$ ) of healthy subject. Here, $n$ indicates the time scale (in beat numbers) over which each measure is calculated. The scaling is obtained using 2 nd order detrended fluctuation analysis, and indicates long-range anticorrelations in the heartbeat interval increment series $\Delta R R_{i}$ [17]. As expected, the scaling properties of the heartbeat interval increment series remain unchanged after the Fourier phase randomization $(\triangle)$. (b) The root mean square fluctuation of the integrated magnitude series $(\square)$ indicates long-range correlations in the magnitude series $\left|\Delta R R_{i}\right|$ (group average exponent of $\alpha-1=0.74 \pm 0.08$ where $F(n) / n \propto n^{\alpha-1}$ ). After Fourier phase randomization of the interbeat interval increment series we find random behavior with exponent $0.5(\triangle)$. This change in the scaling (after removing the nonlinear features in the time series) suggests that the magnitude series carries information about the nonlinear properties of the heartbeat dynamics. (c) The root mean square fluctuation of the integrated sign series ( $\square$ ) indicates anticorrelated behavior in $\operatorname{sign}\left(\Delta R R_{i}\right.$ ) (group average exponent of $\alpha-1=0.42 \pm 0.03$ where $\left.F(n) / n \propto n^{\alpha-1}\right)$. The scaling properties of the sign series remain unchanged after the Fourier phase randomization $(\triangle)$, which suggests that the sign series relates to linear properties of the heartbeat interval time series. We note the apparent crossovers at $n \approx 20$ beats and $n \approx 100$ beats. A gradual loss of anticorrelation in the sign series is observed at time scales larger than $n \approx 100$ beats. The numbers in the figure indicate the scaling exponents before and after the phase-randomization procedure. Adapted from ref. [14]

fluctuation analysis method for estimating the scaling exponents of anticorrelated signals $(\alpha<0.5)$, we integrate the magnitude and sign series; (v) we perform a scaling analysis using 2nd order detrended fluctuation analysis on the integrated magnitude and sign series; (vi) to obtain the scaling exponents for the magnitude and sign series we measure the slope of $F(n) / n$ on a log-log plot, where $F(n)$ is the root mean square fluctuation function and $n$ is the scale of analysis (in beat numbers). 
We find for each subject in a group of 18 healthy individuals [13], that the time series of the magnitudes exhibits correlated behavior (Fig. 2b) (unlike the original heartbeat increment time series, which is anticorrelated, Fig. 2a). The sign series, however, exhibits anticorrelated behavior (Fig. 2c). Correlation in the magnitude series indicates that an increment with large magnitude is more likely to be followed by an increment with large magnitude. Anticorrelation in the sign series indicates that a positive increment is more likely to be followed by a negative increment. Our result for the temporal organization of heartbeat fluctuations thus suggests that, under healthy conditions, a large increment in the positive direction is more likely to be followed by a large increment in the negative direction [14]. We find that this empirical "rule" holds over a broad range of time scales from several up to hundreds of beats (Fig. 2) [15].

To show that fluctuations following an identical scaling law can exhibit different time ordering for the magnitude and sign, we perform a Fourier transform on a heartbeat interval increment time series, preserving the amplitudes of the Fourier transform but randomizing the Fourier phases. Then we perform an inverse Fourier transform to create a surrogate series. This procedure eliminates non-linearities, preserving only the linear features (i.e. two-point correlations) of the original time series [16]. The new surrogate series has the same power spectrum as the original heartbeat interval increment time series, with a scaling exponent indicating long-range anticorrelations in the interbeat increments (Fig. 2a). Our analysis of the sign time series derived from this surrogate signal shows scaling behavior almost identical to the one for the sign series from the original data (Fig. 2c). However, the magnitude time series derived from the surrogate (linearized) signal exhibits uncorrelated behavior - a significant change from the strongly correlated behavior observed for the original magnitude series (Fig. 2b). Thus, the increments in the surrogate series do not follow the empirical "rule" observed for the original heartbeat series, although these increments follow a scaling law identical to the original heartbeat increment series. Moreover, our results raise the interesting possibility that the magnitude series carries information about the nonlinear properties of the heartbeat series, while the sign series relates importantly to linear properties.

We test our analysis on a group of 12 subjects with congestive heart failure [13]. Compared to the healthy subjects, the magnitude exhibits weaker correlations with a scaling exponent closer to the exponent of an uncorrelated series. The change in the magnitude exponent for the heart failure subjects is consistent with a previously reported loss of nonlinearity with disease $[18,19,20,21]$. The sign time series of heart failure subjects shows scaling behavior similar to the one observed in the original time series, but significantly different from the healthy subjects (Table 1).

Next, we investigate how the heart rhythms of healthy subjects change within the different sleep stages. Typically the differences in cardiac dynamics during wake or sleep state, and during different sleep stages are reflected in the average and standard deviation of the interbeat interval time series [22,23]. Recent studies show that changes in cardiac control due to circadian rhythms or different sleep stages can lead to systematic changes in the correlation (scaling) properties of the heartbeat dynamics. In particular it was found that the long-range correlations in heartbeat dynamics change during wake and sleep periods [24], indicating different regimes of intrinsic neuroautonomic regulation of the cardiac dynamics, which may switch on and off with the circadian rhythms.

Healthy sleep consists of cycles of approximately 1-2 hours duration. Each cycle is 
TABLE 1. Comparison of the statistics of the root mean square fluctuation, $F(n)$ (calculated using the 2 nd order detrended fluctuation analysis method $[9,11,12]$ where $n$ is the time scale in beat numbers over which each measure is calculated), and the scaling exponents for 18 healthy subjects and 12 subjects with heart failure [13] (obtained from 6-hour records during the day). The scaling features of the magnitude and sign change significantly for the subjects with heart failure, raising the possibility of bedside applications. $\alpha$ is the best fit to the range $6<n<1024 . F(n)$ is estimated at the crossover position $(n=16)$ (Fig. 2b) where the largest separation between the two groups is estimated. Since we observe two apparent crossovers in the scaling behavior of the sign series, we calculate the scaling exponents in three different regions : (i) the short range regime for time scales $6<n<16$ with scaling exponent $\alpha_{1}$, (ii) the intermediate regime for time scales $16 \leq n \leq 64$ with scaling exponent $\alpha_{2}$, (iii) and the long range regime for time scales $64<n \leq 1024$ with scaling exponent $\alpha_{3}$. For each measure, the group average \pm 1 standard deviation is presented. The values which show highly significant differences ( $p \leq 0.01$ by Student's $t$-test) between the healthy and heart failure groups are indicated in boldface. We note, surprisingly, that the short range and the intermediate range scaling exponents $\alpha_{1}$ and $\alpha_{2}$ of the sign series may provide even more robust separation between healthy and heart failure compared to previous reports [17] based on the scaling exponents of the original heartbeat series.

\begin{tabular}{|c|c|c|c|}
\hline \multicolumn{4}{|c|}{ magnitude } \\
\hline measure & healthy & heart failure & $p$ value \\
\hline $\log _{10} F(n)$ & $-1.49 \pm 0.16$ & $-1.92 \pm 0.17$ & $1 \times 10^{-7}$ \\
\hline$\alpha$ & $1.74 \pm \mathbf{0 . 0 8}$ & $1.66 \pm 0.06$ & 0.01 \\
\hline$\alpha_{1}$ & $1.55 \pm 0.08$ & $1.6 \pm 0.08$ & 0.13 \\
\hline$\alpha_{2}$ & $1.66 \pm 0.08$ & $1.61 \pm 0.08$ & 0.14 \\
\hline$\alpha_{3}$ & $1.82 \pm \mathbf{0 . 1}$ & $1.71 \pm \mathbf{0 . 1}$ & $4 \times 10^{-3}$ \\
\hline \multicolumn{4}{|c|}{ sign } \\
\hline measure & healthy & heart failure & $p$ value \\
\hline $\log _{10} F(n)$ & $\mathbf{0 . 1 4} \pm \mathbf{0 . 0 5}$ & $0.02 \pm 0.06$ & $1 \times 10^{-6}$ \\
\hline$\alpha$ & $1.42 \pm 0.03$ & $1.44 \pm 0.02$ & 0.08 \\
\hline$\alpha_{1}$ & $1.43 \pm 0.12$ & $1.15 \pm 0.12$ & $7 \times 10^{-7}$ \\
\hline$\alpha_{2}$ & $1.27 \pm 0.07$ & $1.41 \pm 0.07$ & $1 \times 10^{-5}$ \\
\hline$\alpha_{3}$ & $1.53 \pm 0.065$ & $1.49 \pm 0.04$ & 0.04 \\
\hline
\end{tabular}

characterized by a sequence of sleep stages usually starting with light sleep, followed by deep sleep, and rapid eye movement (REM) sleep [25]. While the specific functions of the different sleep stages are not yet well understood, many believe that deep sleep is essential for physical rest, while REM sleep is important for memory consolidation [25]. Different sleep stages during nocturnal sleep were found to relate to a specific type of correlations in the heartbeat intervals [26], suggesting a change in the mechanism of cardiac regulation in the process of sleep.

We considered 24 records of interbeat intervals obtained from 12 healthy individuals during sleep [28]. The records have an approximate duration of 7.5 hours. The anno- 


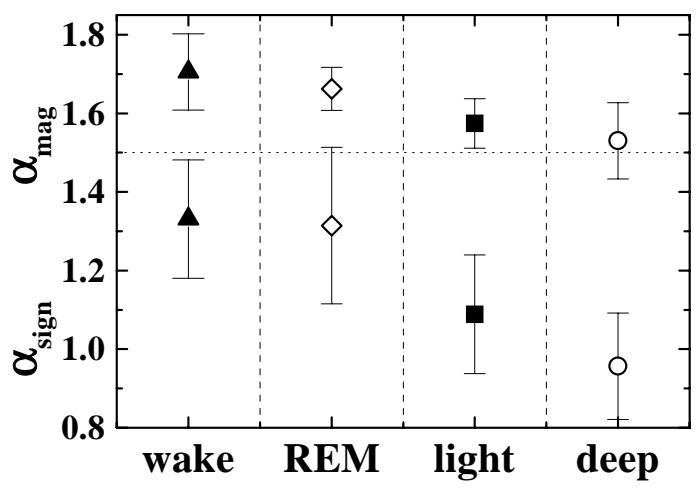

FIGURE 3. The average values of the fluctuation exponents $\alpha_{\text {mag }}$ for the magnitude series and $\alpha_{\text {sign }}$ for the sign series for the different phases (wake state, REM sleep, light sleep, and deep sleep). For each of the 24 records from 12 healthy subjects the corresponding 2nd order DFA fluctuation functions $F(n)$ have been fit in the range of $8 \leq n \leq 13$ and $11 \leq n \leq 150$ heartbeats for $\alpha_{\text {sign }}$ and $\alpha_{\text {mag }}$, respectively, where the most significant differences between the sleep stages occur. After ref. [28]

tation and duration of the sleep stages were determined based on standard procedures [27]. We applied the detrended fluctuation analysis (DFA) method $[9,10,11,12]$ on both the sign and the magnitude time series. We found that the sign series exhibits anticorrelated behavior at short time scales which is characterized by a correlation exponent with smallest value for deep sleep, larger value for light sleep, and largest value for REM sleep. The magnitude series, on the other hand, exhibits uncorrelated behavior for deep sleep, and long-range correlations are found for light and REM sleep, with a larger exponent for REM sleep. The observed increase in the values of both the sign and magnitude correlation exponents from deep through light to REM sleep is systematic and significant. We also found that the values of the sign and magnitude exponents for REM sleep are very close to the values of these exponents for the wake state [28].

The mean values and their standard deviations for the different sleep stages are shown in Fig. 3. We estimate the exponents $\alpha$ from the slopes in the log-log plot of $F(n)$ versus $n$ for all records. Since the most significant differences for the short-range sign correlations occur in the range of $8 \leq n \leq 13$ heartbeats, we use this fitting range for the exponents $\alpha_{\text {sign }}$. For the magnitude exponent $\alpha_{\text {mag }}$, we use the range $11 \leq n \leq 150$, since the long-range correlations occurring in light and REM sleep can be observed best in this region. We find that there is a significant difference in the sign series exponent $\alpha_{\text {sign }}$ observed for all three sleep stages (the $p$-value, obtained by the Student's $t$-test, is below 0.001). The magnitude correlation exponents for REM sleep and for intermediate wake states are significantly larger than those for the non-REM stages (light and deep sleep). Here also, the $p$-values are less than 0.001 . Note that we do not find a significant difference between the average exponents for REM sleep and for the intermediate wake states. This is not surprising because heartbeat activity during REM sleep is very close to heartbeat activity during the wake state and the heartbeat time series during REM and wake exhibit similar scaling properties $[24,26]$

More significant than the differences for the average exponents are the differences 


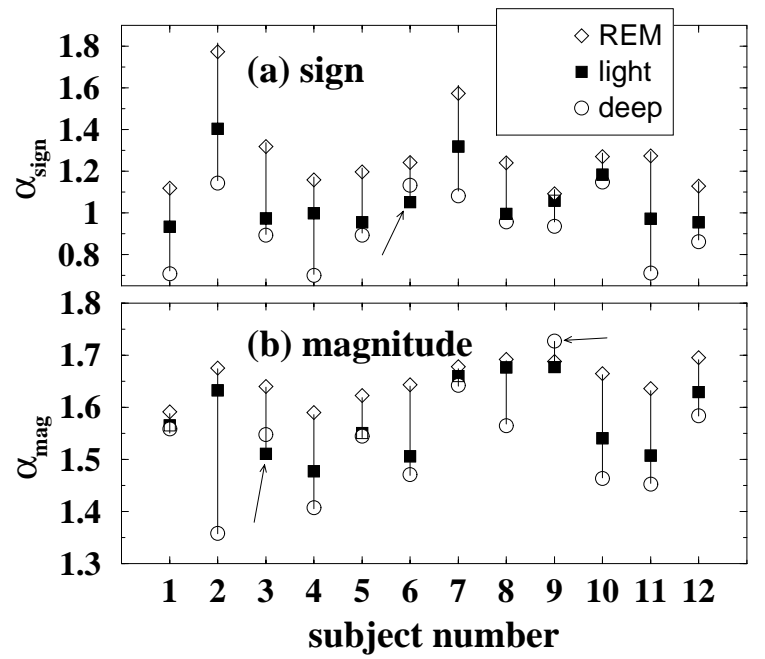

FIGURE 4. The values of the effective fluctuation exponents $\alpha$ for the integrated sign series (a) and the integrated magnitude series (b) are shown for all 12 healthy subjects (second night of recording). While the $\alpha$ values are fluctuating, for REM sleep the $\alpha$ is larger than the $\alpha$ for light sleep, which is larger than the $\alpha$ for deep sleep (the 3 arrows indicate the cases which are not ordered in the same way as the majority). The exponent values have been determined over the fitting ranges as described in the caption of Fig. 3. After ref. [28].

between the exponents for each individual. Figure 4 shows the $\alpha$ values for REM, light, and deep sleep for all 12 healthy subjects (second night only). In almost all cases the exponent of the REM sleep is the largest, the exponent of the light sleep is intermediate, and the exponent of the deep sleep is smallest (there are three exceptions, indicated by arrows). In our group of 24 records from 12 healthy individuals, we find larger exponents in REM sleep than in deep sleep for $100 \%$ of the sign series and for $88 \%$ of the magnitude series.

Our results suggest that the empirical "rule" mentioned above also applies to REM sleep, while in deep sleep small and large increments seem to appear in a random fashion. On the other hand, the stronger sign anticorrelations in deep sleep indicate that a positive increment is more likely - even more likely than in REM sleep - to be followed by a negative increment. Thus, the correlation behavior of the heartbeat increments and their signs and magnitudes during daytime activity is similar to the behavior we find in REM sleep, but quite different from the behavior we observe in deep sleep. This is consistent with our finding [Fig. 3] of average exponent values for the wake episodes similar to the exponent values for REM sleep.

We conclude that series with identical correlation properties can have completely different time ordering which can be characterized by different scaling exponents for the magnitude and sign series. Moreover, we show that the magnitude series carries information regarding the nonlinear properties of the original series while the sign series carries information regarding the linear properties of the original series. The significant 
decrease in the short-range scaling exponent for the sign series in heart failure may be related to perturbed vagal control affecting relatively high frequency fluctuations. The decrease of the long-range scaling exponent for the magnitude series of the heart failure patients indicates weaker correlations and loss of nonlinearity which may be related to impaired feedback mechanisms of neurohormonal cardiac regulation. Further we observe short-range anticorrelations in the sign of the interbeat interval increments which are stronger during deep sleep, weaker during light sleep, and even weaker during REM sleep. In contrast, the magnitude of the increments is long-range correlated with a larger exponent during REM sleep suggesting stronger nonlinear contributions to the heartbeat dynamics in this stage compared with weaker nonlinear contributions in the non-REM stages.

\section{ACKNOWLEDGMENTS}

We thank A. Bunde, A.L. Goldberger, S. Havlin, C.-K. Peng, and T. Penzel for discussions and major contributions to the results reviewed here which represent collaborative research efforts. We thank the NIH/National Center for Research Resources (P41 RR13622), the Israel-U.S.A Binational Science Foundation and the Deutscher Akademischer Austauschdienst (DAAD) for support. The healthy volunteers were recorded as part of the SIESTA project funded by the European Union grant no. Biomed-2-BMH4-CT97-2040.

\section{REFERENCES}

1. M.F. Shlesinger, Ann. NY Acad. Sci. 504, 214 (1987).

2. T. Vicsek, Fractal Growth Phenomenon 2nd edn (World Scientific, Singapure, 1993).

3. H. Takayasu, Fractals in the Physical Sciences (Manchester Univ. Press, Manchester, UK, 1997).

4. R.M. Berne and M.N. Levy, Cardiovascular Physiology, 7th edn (Mosby, St. Louis, 1996).

5. M. Kobayashi and T. Musha, IEEE Trans. Biomed. Eng. 29, 456 (1982).

6. P.Ch. Ivanov, M.G. Rosenblum, C-K Peng, J. Mietus, S. Havlin, H.E. Stanley, and A.L. Goldberger, Nature 383, 323 (1996).

7. P.Ch. Ivanov, L.A.N. Amaral, A.L. Goldberger, S. Havlin, M.G. Rosenblum, H.E. Stanley, Z. Struzik, Chaos 11, 641 (2001).

8. C.-K. Peng, J. Mietus, J. M. Hausdorff, S. Havlin, H. E. Stanley, and A. L. Goldberger, Physical Review Letters 70, 1343 (1993).

9. C.-K. Peng, S.V. Buldyrev, S. Havlin, M. Simons, H.E. Stanley, and A.L. Goldberger, Physical Review E 49, 1685 (1994).

10. J.W. Kantelhardt, E. Koscielny-Bunde, H.H.A. Rego, S. Havlin, and A. Bunde, Physica A 294, 441 (2001).

11. K. Hu, P.Ch. Ivanov, Z. Chen, P. Carpena, and H.E. Stanley, Physical Review E 64, 011114(19) (2001).

12. Z. Chen, P.Ch. Ivanov, K. Hu, and H.E. Stanley, Physical Review E 65, 041107(15) (2002).

13. MIT-BIH Normal Sinus Rhythm Database and BIDMC Congestive Heart Failure Database available at http://www.physionet.org/physiobank/database/\#ecg.

14. Y. Ashkenazy, P.Ch. Ivanov, S. Havlin, C.-K. Peng, A.L. Goldberger, and H.E. Stanley, Physical Review Letters 86, 1900 (2001).

15. Heartbeat increment series were investigated by A. Babloyantz and P. Maurer, Phys. Lett. A 221, 43 (1996) and P. Maurer, H.-D. Wang, and A. Babloyantz, Phys. Rev. E 56, 1188 (1997). These studies 
differ from ours because we investigate, quantitatively, normal heartbeats by evaluating the scaling properties of the magnitude and sign series. In addition, our calculations are based on window scales larger than 6 and up to one thousand heartbeats.

16. P.F. Panter, Modulation, Noise, and Spectral Analysis Applied to Information Transmission (New York, New York, 1965). We also applied a test for nonlinearity using the phase randomization procedure described in J. Theiler, S. Eubank, A. Longtin, B. Galdrikian, and D.J. Farmer, Physica D 58, 77 (1992), and find that the magnitude scaling exponent drops to 0.5 while the sign scaling exponent remains unchanged.

17. C.-K. Peng, S. Havlin, H.E. Stanley, and A.L. Goldberger, Chaos 5, 82 (1995).

18. J. Kurths, A. Voss, P. Saparin, A. Witt, H.J. Kleiner, and N. Wessel, Chaos 5, 88 (1995).

19. G. Sugihara, W. Allan, D. Sobel, and K.D. Allan, Proc. Natl. Acad. Sci. USA 93, 2608 (1996).

20. P.Ch. Ivanov, M.G. Rosenblum, L.A.N. Amaral, Z.R. Struzik, S. Havlin, A.L. Goldberger, and H.E. Stanley, Nature 399, 461 (1999).

21. L.A.N. Amaral, P.Ch. Ivanov, N. Aoyagi, I. Hidaka, S. Tomono, A.L. Goldberger, H.E. Stanley, and Y. Yamamoto. Physical Review Letters 86, 6026 (2001).

22. H. Moelgaard, K. E. Soerensen, and P. Bjerregaard, Am. J. Cardiology 68, 77 (1991).

23. H. V. Huikuri, K. M. Kessler, E. Terracall, A. Castellanos, M. K. Linnaluoto, and R. J. Myerburg, Am. J. Cardiology 65, 391 (1990).

24. P. Ch. Ivanov, A. Bunde, L. A. N. Amaral, S. Havlin, J. Fritsch-Yelle, R. M. Baevsky, H. E. Stanley, and A. L. Goldberger, Europhysics Letters 48, 594 (1999).

25. M. A. Carskadon and W. C. Dement, in: Principles and Practice of Sleep Medicine, edited by M. H. Kryger, T. Roth, and W. C. Dement (W. B. Saunders, Philadelphia, 1994), pp. 16-25.

26. A. Bunde, S. Havlin, J. W. Kantelhardt, T. Penzel, J.-H. Peter, and K. Voigt, Physical Review Letters 85, $3736(2000)$.

27. A. Rechtschaffen and A. Kales, A Manual of Standardized Terminology, Techniques, and Scoring System for Sleep Stages of Human Subjects (U.S. Government Printing Office, Washington, 1968).

28. J.W. Kantelhardt, Y. Ashkenazy, P.Ch. Ivanov, A. Bunde, S. Havlin, T. Penzel, J.-H. Peter, and H.E. Stanley, Physical Review E 65, 051908(6) (2002). 